\title{
Associated Risks of Obesity and/or Metabolic Syndrome with Intra-operative or Post-Operative Occurrences in Patients with Open Ventral Hernia Repair
}

\author{
Maytham Hameed Al-Qanbar ${ }^{1,2}$ \\ ${ }^{1}$ Department of Surgery College of Medicine University of Kufa, Najaf, Iraq \\ ${ }^{2}$ General Surgery Department, AlSader Teaching Hospital, Najaf, Iraq
}

Email address:

mhkenber@yahoo.com

To cite this article:

Maytham Hameed Al-Qanbar. Associated Risks of Obesity and/or Metabolic Syndrome with Intra-operative or Post-Operative Occurrences in Patients with Open Ventral Hernia Repair. Journal of Surgery. Vol. 5, No. 4, 2017 pp. 62-67. doi: 10.11648/j.js.20170504.12

Received: June 15, 2017; Accepted: July 3, 2017; Published: August 3, 2017

\begin{abstract}
Metabolic syndrome and obesity are increasing in incidence worldwide and they carry with them a lot of health issues that might directly affect individuals or might be associated with higher risk of surgical complications represented in this study by open ventral hernia repair. A cohort study of 132 patients admitted electively for open ventral hernia repair to the surgical wards at Al-Sader teaching hospital in Najaf /Iraq from December 1st/ 2015 to October 1st/ 2016. All patients were followed for intra-operative occurrences and followed for 2 weeks postoperatively for any early post-operative complications. Of the total 132 patients, there were 44 patients (33.3\%) having metabolic syndrome and there were 68 obese patients $(66.6 \%)$ of which 37 having metabolic syndrome (54.4\%). There was no significant association between intra-operative occurrences or early post-operative complications in obese patients with or without metabolic syndrome but there was a significant association between intra-operative occurrences and metabolic syndrome in patients having body mass index (BMI) below $30 \mathrm{Kg} / \mathrm{m}^{2}$. Diabetes was the only significant factor for early postoperative wound complications whether the patient had metabolic syndrome or not.
\end{abstract}

Keywords: Ventral Hernia, Metabolic Syndrome, Post-Operative, Intra-operative, Complications, Obese, BMI

\section{Introduction}

A ventral hernia can be defined as a protrusion of viscera through a defect or a hole in the anterior abdominal wall. This term includes epigastric, para-umbilical, umbilical and incisional hernias. $[1,2]$ Repairs of such hernias are done annually in 348000 patients in USA and 300000 patients in Europe [3].

Obesity and metabolic syndrome (MS) on the other hand are rising health problems variably affecting different societies like $35 \%$ of USA population, $8.4 \%$ of Japanese population and $24.5 \%$ of Chinese population are affected with MS. [4] Body mass index (BMI) or Quetelet index is defined as the body mass divided by the square of the body height in meters $\left(\mathrm{kg} / \mathrm{m}^{2}\right)$ and depending on this index the WHO regards a BMI of less than 18.5 as underweight, while a BMI equal to or greater than 25 is considered overweight and above 30 is considered obese [5]. According to the
American Heart Association (AHA), metabolic syndrome is diagnosed when a patient has at least 3 of the following 5 conditions: fasting glucose $\geq 100 \mathrm{mg} / \mathrm{dL}$ (or receiving drug therapy for hyperglycemia), blood pressure $\geq 130 / 85 \mathrm{~mm} \mathrm{Hg}$ (or receiving drug therapy for hypertension), triglycerides $\geq$ $150 \mathrm{mg} / \mathrm{dL}$ (or receiving drug therapy for hypertriglyceridemia), HDL-C $<40 \mathrm{mg} / \mathrm{dL}$ in men or $<50$ $\mathrm{mg} / \mathrm{dL}$ in women (or receiving drug therapy for reduced HDL-C) and waist circumference $\geq 102 \mathrm{~cm}$ (40 in) in men or $\geq 88 \mathrm{~cm}$ (35 in) in women. The cardinal features of MS are abnormal fat deposition and function which arise from insulin resistance [6]. Inflammation and oxidative stress accompanying adipocytes hypertrophy secondary to surplus of fat storage lead to excess release of proinflammatory cytokines like interleukin-1 (IL-1), interleukin-6 (IL-6) (one third of total IL-6 concentration come from adipose tissue), 
C-reactive protein and tumor-necrosis factor alpha (TNF- $\alpha$ ), resulting in low-grade chronic inflammation, which begins in adipose tissue and eventually reaches the circulation and other organs. [7]

Since MS pathogenesis theory involves release and over expression of above mentioned pro-inflammatory cytokines (IL-1, IL-6, TNF- $\alpha$ and CRP) which also paly important role in local and systemic inflammatory response to injury, a hypothesis has been made that there might be some influence of these changes on the intra-operative body response to surgical trauma or on the development of early postoperative wound complications during the process of healing.

\section{Patients and Method}

\subsection{Study Design}

A cohort study of patients with ventral hernia for assessment of the risk of perioperative complications for those who were obese with MS, those who were obese without MS compared to those who were non-obese with non-metabolic syndrome in order to assess the obesity and metabolic syndrome as risk factors for perioperative morbidity.

\subsection{Patients Selection}

All patients with ventral hernia whom were admitted for open repair at Al Sader medical city in Al-Najaf Al-Ashraf province from $1^{\text {st }}$ of December 2015 to $1^{\text {st }}$ of October 2016 were included in the study. All were admitted for elective surgical repair (obstructed type were excluded) under general anesthesia. All patients were well prepared and fit for surgery regarding their medical conditions.

Preoperative measurements and laboratory results included age, height, weight, waist circumference, blood pressure (BP), fasting blood sugar (FBS), HbAlc, serum cholesterol, serum triglyceride, serum low density lipoprotein (S. LDL) and serum high density lipoprotein (S. HDL); in addition to medical history about their illness duration and medication used (antihypertensive, hypoglycemic agents, lipid lowering agents, and duration of these medications).

\subsection{Recorded Occurrences}

Intraoperatively, the below mentioned parameters were recorded if they needed immediate action from the anesthetist to correct them (like increase $\mathrm{O} 2$ flow rate, decrease ventilator pressure, atropine injection. etc). These include: decrease in $\mathrm{O} 2$ saturation (i.e. Desaturation which was defined as oxygen saturation $<$ or $=90 \%$ for $>$ or $=10$ seconds), tachycardia ( $>100 \mathrm{BPM})$, bradycardia $(<60 \mathrm{BPM})$, decrease in BP $(<110 / 60)$ and increase in BP $(>140 / 90)$. Postoperative recovery was classified into smooth, delayed or ICU admission.

Patients were followed for around 14 days postoperatively (i.e. until removal of stitches).

Complications searched for postoperatively were:

1-Atelectasis 2-Bleeding (hematoma) 3-Pulmonary embolism 4-Seroma 5-Wound infection 6-DVT

\subsection{Statistical Analysis}

Statistical analysis was done using SPSS (statistical package for social sciences) version 20 in which we use number and percentage as descriptive statistics. Relative risk with $95 \%$ confidence interval (CI) was computed to measure risk of exposure to certain risk factors. $\mathrm{P}$ value of $\leq 0.05$ regarded as significant.

\section{Results}

A total of 132 patients with ventral hernia were included in this study. The mean age of patients with MS was $46.2 \pm$ 11.6 years while those without MS was $41.1 \pm 10.1$ years ( $p$ $=0.011)$.

Table 1 shows the basic characteristics of the patients involved in this study and although it showed female preponderance but this was statistically insignificant $(\mathrm{p}=$ 0.564). Other parameters in the table were used to categorize patients into those with metabolic syndrome and those who were not, blood pressure readings, FBS, TG, HDL, waist circumference, BMI, type of hernia repair regarding mesh usage or not.

Table 1. Basic characteristics of patients of the study group.

\begin{tabular}{llll}
\hline & & Frequency & Percent \\
\hline \multirow{2}{*}{ Gender } & Male & 35 & 26.5 \\
& Female & 97 & 73.5 \\
& $\geq 102$ male & 25 & 18.9 \\
Waist circumference $\mathrm{cm}$ & $\geq 88$ female & 83 & 62.9 \\
& $<102$ male & 10 & 7.6 \\
& $<88$ female & 14 & 10.6 \\
Blood pressure mmHg & hypertension & 29 & 22.0 \\
\multirow{2}{*}{ Fasting blood sugar mg/dl } & normotensive & 103 & 78.0 \\
& hyperglycemia & 22 & 16.7 \\
Triglyceride mg/dl & not diabetic & 110 & 83.3 \\
& elevated & 30 & 22.7 \\
HDL $\mathrm{mg} / \mathrm{dl}$ & normal & 102 & 77.3 \\
\hline
\end{tabular}




\begin{tabular}{llll}
\hline & & Frequency & Percent \\
\hline \multirow{2}{*}{ BMI kg/m2 } & $\geq 30$ & 68 & 51.5 \\
& $<30$ & 64 & 48.5 \\
Type of repair & Hernioplasty & 121 & 91.7 \\
& Hernioraphy & 11 & 8.3 \\
Metabolic syndrome & with & 44 & 33.3 \\
& without & 88 & 66.7 \\
\hline
\end{tabular}

There were 68 patients $(66.6 \%)$ with $\mathrm{BMI} \geq 30$ of which $37(54.4 \%)$ had metabolic syndrome while 31 did not have MS. Hypotension was the most common intra-operative occurrence $(13.5 \%)$ reported in patients with metabolic syndrome with $\mathrm{BMI} \geq 30$ followed by hypertension $(8.1 \%)$ and the least were bradycardia and decrease $\mathrm{O} 2$ saturation both of which was $2.7 \%$. While hypertension was the main occurrence in the other group (BMI $30 \geq$ and no metabolic syndrome). The rate of wound infection, seroma and other post-operative complications were recorded according to this sub-classification with their relative risk (RR) assessment. Other complications such as deep veins thrombosis, pulmonary embolism or wound dehiscence are not present in the table because they were not occurred in any of our patients. Both of these groups' findings were not directly related to metabolic syndrome as evident by the value of $95 \%$ confidence interval of the relative risks shown in table 2 .

Table 2. Complications among patients with BMI $\geq 30$ and metabolic syndrome as risk factor.

\begin{tabular}{llll}
\hline Occurrence & MS $(\mathbf{N}=\mathbf{3 7})$ & NOMS (N= 31) & RR (95\% CI) \\
\hline Intraoperative & & & $0.83(0.02-30.4)$ \\
Bradycardia & $1(2.7 \%)$ & $1(3.2 \%)$ & $0.44(0.016-6.1)$ \\
Tachycardia & $2(5.4 \%)$ & $1(3.2 \%)$ & $0.31(0.06-1.182)$ \\
Hypertension & $3(8.1 \%)$ & $8(25.8 \%)$ & $1.39(0.3-7.1)$ \\
Hypotension & $5(13.5 \%)$ & $3(9.6 \%)$ & $0.83(0.02-30.4)$ \\
Desaturation & $1(2.7 \%)$ & $1(3.2 \%)$ & $0.55(0.06-3.9)$ \\
Delayed recovery & $3(8.1 \%)$ & $2(6.4 \%)$ & $1.39(0.31-7.13)$ \\
Postoperative & & & $3.07(0.89-13.41)$ \\
Seroma & $5(13.5 \%)$ & $3(9.6 \%)$ & $0.83(0.08-8.15)$ \\
Wound infection & $11(29.7 \%)$ & $3(9.6 \%)$ & $0.41(0.015-5.7)$ \\
Atelectasis & $2(5.4 \%)$ & $2(6.4 \%)$ & $2(6.4 \%)$ \\
Hematoma & $1(2.7 \%)$ & & \\
\hline
\end{tabular}

Metabolic syndrome was reported in 44 patients in this study; 37 of whom were obese (i.e. with BMI $\geq 30$ ) and 7 were not. The effect of obesity as a risk factor for development of intra or post-operative occurrences is studied in table (3) which showed no risk association (of obesity) with such occurrences as evident by the relative risk of each occurrence. Although 11 patients (29.7\%) with BMI $\geq 30$ had wound infection whereas $2(28.5 \%)$ patients with $\mathrm{BMI}<30$ had wound infection (beside other complications shown in the same table); the effect of obesity per se as a risk factor for development of these complications was not apparent as evident by the $95 \%$ confidence interval.

Table 3. Complications among patients with metabolic syndrome.

\begin{tabular}{llll}
\hline Occurrence & BMI $\geq \mathbf{3 0}(\mathbf{n}=\mathbf{3 7})$ & BMI $<\mathbf{3 0}(\mathbf{n}=\mathbf{7})$ & RR $(\mathbf{9 5} \% \mathbf{C I})$ \\
\hline Intraoperative & & & $0.18(0.005-6.8)$ \\
Bradycardia & $1(2.7 \%)$ & $1(14.3 \%)$ & $0.4(0.008-3118823)$ \\
Tachycardia & $1(2.7 \%)$ & $0(0 \%)$ & $3.24(0.32-1806667)$ \\
Hypertension & $8(21.6 \%)$ & $0(0 \%)$ & $0.94(0.13-21.2)$ \\
Hypotension & $5(13.5 \%)$ & $1(14.3 \%)$ & $0.4(0.008-3118823)$ \\
Desaturation & $1(2.7 \%)$ & $0(0 \%)$ & $0.81(0.03-531372)$ \\
Delayed recovery & $2(5.4 \%)$ & $0(0 \%)$ & $0.81(0.039-531372)$ \\
postoperative & & & $0.09(0.003-1.26)$ \\
Atelectasis & $2(5.4 \%)$ & $0(0 \%)$ & $1.48(0.13-857281)$ \\
Hematoma & $1(2.7 \%)$ & $2(28.5 \%)$ & $1.041(0.33-6.5)$ \\
Seroma & $5(13.5 \%)$ & $0(0 \%)$ & $2(28.5 \%)$ \\
Wound infection & $11(29.7 \%)$ & & \\
\hline
\end{tabular}

A total of 88 patients in this study were not having metabolic syndrome of which 31 were obese and 57 were not. There was no any significant association between intra or post-operative occurrences and obesity in those who were not having metabolic syndrome (table 4). 
Table 4. Occurrences among patients without metabolic syndrome and obesity as a risk factor.

\begin{tabular}{llll}
\hline Occurrence & BMI $\geq \mathbf{3 0}(\mathbf{n}=\mathbf{3 1})$ & $\mathbf{B M I}<\mathbf{3 0}(\mathbf{n}=\mathbf{5 7})$ & $\mathbf{R R}(\mathbf{9 5} \% \mathbf{C I})$ \\
\hline Intraoperative & & & $0.61(0.025-6.37)$ \\
Bradycardia & $1(3.2 \%)$ & $3(5.3 \%)$ & $7.41(0.34-\mathrm{inf})$ \\
Tachycardia & $2(6.4 \%)$ & $0(0 \%)$ & $5.5(0.53-137.2)$ \\
Hypertension & $3(9.6 \%)$ & $1(1.7 \%)$ & $0.91(0.18-3.87)$ \\
Hypotension & $3(9.6 \%)$ & $6(10.5 \%)$ & $0.9(0.03-12.6)$ \\
Desaturation & $1(3.2 \%)$ & $2(3.4 \%)$ & $1.83(0.3-11.06)$ \\
Delayed recovery & $3(9.6 \%)$ & $3(5.3 \%)$ & $1.22(0.146-8.72)$ \\
Postoperative & & & $7.4(0.34-4862346)$ \\
Atelectasis & $2(6.4 \%)$ & $3(5.3 \%)$ & $1.83(0.3-11)$ \\
Hematoma & $2(6.4 \%)$ & $0(0 \%)$ & $0.78(0.66-3.13)$ \\
Seroma & $3(9.6 \%)$ & $3(5.3 \%)$ & $7(12.3 \%)$ \\
Wound infection & $3(9.6 \%)$ & & \\
\hline
\end{tabular}

There was significant association between intraoperative occurrences and those who were nonobese with metabolic syndrome except for hypertension (table 5).

Table 5. Intraoperative complications among patients with $B M I<30$ and metabolic syndrome the risk factor.

\begin{tabular}{llll}
\hline Occurrence & MS (N = 7) & NO MS (N = 57) & RR (95\%CI) \\
\hline Bradycardia & $3(42.8 \%)$ & $1(1.7 \%)$ & $24.4(2.49-600)$ \\
Hypertension & $1(14.3 \%)$ & $0(0 \%)$ & $16.4(0.3-$ inf.) \\
Hypotension & $6(85.7 \%)$ & $1(1.7 \%)$ & $48.8(7.6-634.4)$ \\
Desaturation & $2(28.5 \%)$ & $0(0 \%)$ & $32.8(1.5$-inf $)$ \\
$\begin{array}{l}\text { Delayed } \\
\text { recovery }\end{array}$ & $3(42.8 \%)$ & $0(0 \%)$ & $49.2(3.1-$ inf. $)$ \\
\hline
\end{tabular}

In order to assess diabetes mellitus as a risk factor for postoperative complications a part from metabolic syndrome component patient were classified into diabetics (25 patients) and nondiabetics (107 patients). There was only significant association of diabetes with wound infection with a relative risk of 8.02 with a $95 \%$ confidence interval of $3.67-17.42$ otherwise there was no any significant association between diabetes and other post-operative complications (table 6).

Table 6. Postoperative complications among diabetics and non-diabetics.

\begin{tabular}{llll}
\hline Complication & $\begin{array}{l}\text { Diabetics } \\
(\mathbf{n = 2 5 )}\end{array}$ & $\begin{array}{l}\text { Non diabetic } \\
(\mathbf{n = 1 0 7})\end{array}$ & RR (95\% CI) \\
\hline Atelectasis & $0(0 \%)$ & $7(6.5 \%)$ & $0.3(0-3.77)$ \\
Hematoma & $1(4 \%)$ & $4(3.7 \%)$ & $1.07(0.04-9.65)$ \\
Seroma & $3(12 \%)$ & $8(7.5 \%)$ & $1.43(0.3-5.48)$ \\
$\begin{array}{l}\text { Wound } \\
\text { infection }\end{array}$ & $15(60 \%)$ & $8(7.5 \%)$ & $8.02(3.67-17.42)$ \\
\hline
\end{tabular}

Mesh application was also assessed as a risk factor for development of post-operative wound infection. Although the rate of infection among those with metabolic syndrome and mesh repair was $26.3 \%$ and $12 \%$ in those without metabolic syndrome this result was insignificant as cleared by the odd ratios shown in table 7 and table 8 .

Table 7. The relation between type of repair and early postoperative complications in those with metabolic syndrome group.

\begin{tabular}{llll}
\hline Complication & hernioplasty $(\mathbf{n}=\mathbf{3 8})$ & herniorraphy $(\mathbf{n}=\mathbf{6})$ & RR (95\% CI) \\
\hline Atelectasis & $2(5.3 \%)$ & $0(0 \%)$ & $0.684(0.03$-inf.) \\
Hematoma & $3(7.9 \%)$ & $0(0 \%)$ & $1.026(0.068$-inf. $)$ \\
Seroma & $5(13.2 \%)$ & $0(0 \%)$ & $1.7(0.15$-inf.) \\
Wound infection & $10(26.3 \%)$ & $3(50 \%)$ & $0.526(0.24-2.18)$ \\
\hline
\end{tabular}

Table 8. The relation between type of repair and early postoperative complications in those without metabolic syndrome group.

\begin{tabular}{llll}
\hline Complication & hernioplasty $(\mathbf{n}=\mathbf{8 3})$ & herniorraphy $(\mathbf{n}=\mathbf{5})$ & RR $(\mathbf{9 5} \% \mathbf{C I})$ \\
\hline Atelectasis & $5(6 \%)$ & $0(0 \%)$ & $0.66(0.06$-inf. $)$ \\
Hematoma & $2(2.4 \%)$ & $0(0 \%)$ & $0.26(0.013$-inf. $)$ \\
Seroma & $6(7.2 \%)$ & $0(0 \%)$ & $0.795(0.08$-inf. $)$ \\
Wound infection & $10(12 \%)$ & $0(0 \%)$ & $1.32(0.15$-inf. $)$ \\
\hline
\end{tabular}

The most common post-operative complication was wound infection representing a total of 23 out of 132 cases (17.4\%) and for risk stratification we used logistic regression to estimate if there was a significant association between different risk factors (obesity, metabolic syndrome, use of mesh and being diabetic) and occurrence of wound infection which revealed that such association is only present in diabetic patients (table 9).

Table 9. Logistic regression for different risk factors for wound infection.

\begin{tabular}{lllll}
\hline & P value & \multirow{2}{*}{ OR } & 95\% C. I. for OR & Upper \\
\cline { 4 - 5 } & & Lower & 61.309 \\
DM & .000 & 18.914 & 5.835 & 9.166 \\
Metabolic syndrome & .106 & 2.723 & .809 & 5.824 \\
repair & .918 & .907 & .141 & 1.106 \\
BMI $\geq 30$ & .065 & .199 & .036 & \\
\hline
\end{tabular}




\section{Discussion}

The patients were assigned according to the parameters into those with metabolic syndrome and those who were not; the mean age for metabolic syndrome group was $46.2 \pm 11.6$ year while for the non-metabolic syndrome group was $41.1 \pm$ 10.1 year $(\mathrm{p}=0.011)$ which was a significant difference and this is consistent with the fact that metabolic syndrome prevalence increase with an increased age [8]. This finding indirectly reflects that there was a proper characterization of patients with metabolic syndrome versus those who do not have it.

For intraoperative occurrences among patients with BMI > 30 , there was no significant effect of metabolic syndrome or obesity (tables 2, 3 and 4) on their occurrence in contradiction to Uakritdathikarn et al (2008) who found significant association between obesity and desaturation. [9] This is may be due to the difference in the cut off reading of $\mathrm{SP} \mathrm{O}_{2}$ in their study (which was equal or below $95 \%$ for 10 seconds) and we selected the cut off value of $90 \%$ (because the alarm of the ventilators for SP $\mathrm{O}_{2}$ is pre-set to this value by the factory). On the contrary there was a significant effect of metabolic syndrome on rate of intraoperative occurrences (except for hypertension) in the group with BMI below 30 (table 5). This is paradoxical to expectation but this could be in part attributed to more close-up monitoring of the obese patients which aid in prevention of such complications and in part due to small number of this group of patients (only 7). We did not find any study discuss the influence of the metabolic syndrome on patients with ventral hernia repair intra-operatively (for sake of comparison).

Wound infection was the most common postoperative complication in those with a BMI $>30$ which occurred in 14 patients; $11(29.7 \%)$ in those with metabolic syndrome and 3 $(9.6 \%)$ in those without metabolic syndrome. Although there was relatively big difference in the rate of infection of both groups, the difference was statistically insignificant (RR 3.07; 95\% CI 0.89- 13.41) which would exclude metabolic syndrome per se as a risk factor for wound infection in such cases.

Seroma was the next common complication in the list, occurred in $5(13.5 \%)$, patients with metabolic syndrome and in $3(9.6 \%)$ patients without metabolic syndrome RR $1.39 \mathrm{CI}$ (0.31-7.13) followed by atelectasis and hematoma all of which has neither significant correlation with BMI nor with metabolic syndrome as evident by their relative risks at $95 \%$ confidence interval shown in table (2) and (3). This result was not fully in accordance with the study held by Krpata etal (2012) which showed increased significance of surgical site occurrence (SSO) which occurred in $16 \%$ of the eighty eight patients involved in the study (wound infection $12.5 \%$, seroma $2.27 \%$ and wound dehiscence $1.13 \%$ ) in patients with ventral hernia repair who had co-morbidities like obesity, diabetes, chronic obstructive pulmonary disease and immune suppression ( $\mathrm{p}$ value of 0.02 ); in our study only diabetes was significantly associated with SSO. Krpata did not specify the metabolic syndrome per se as a risk factor. [10]

Results in this study did not show an effect of obesity by itself or as part of metabolic syndrome on patients with ventral hernia repair, where as other studies demonstrated a significant effect of obesity and associated comorbidities on outcome of patients undergoing repaired ventral hernia [10, $11,12]$. This discrepancy may be in part due to the period of follow up of their patients was much longer and not confined to the early postoperative period and in part due to differences in the characteristics of patient involved in their studies like patients with obstructed henias or patients with multiple recurrences also which might add to the risk of such complications.

Other postoperative complications although their occurrence were statistically insignificant to be associated with obesity or metabolic syndrome they were comparable to Fekkes et al (2015) study. [13] Patients in his study developed seroma in $9.6 \%$, hematoma in $6.4 \%$ and atelectasis in $6.4 \%$ which is comparable to our results (tables 2 and 4 ).

Wound infection reported in 20 cases $(16.5 \%)$ out of 121 hernioplasty repairs and in 3 cases (2.47) of 11 herniorrhaphy type of repair. This difference was statistically insignificant after performing logistic regression for risk stratification for estimating mesh insertion as a risk factor for wound infection (table 9). This finding differs from that of Robinson et al (2005) [14] who showed $42 \%$ incidence of mesh related infection which is a very high rate (the rate of mesh related infection was variable according to the study like Lledó et al [12] 1.3\%, Krpata [9] 12.5\% and Falagas 8\% [15] although he studied the rate of infection according to the type of mesh used (whether they are natural or synthetic, absorbable or not. etc) and did not take any other factor in consideration while in our study we just used a single type of mesh material which was poly propylene. On the other hand use of mesh was not associated with increased risk of infection when used to reinforce wound closure to decrease the incidence of incisional hernia occurrence as shown by Wang et al (2016) [16].

Only diabetes was significantly associated with surgical site infection in this study a finding which is in accordance with a lot of studies $[10,11,12]$. Diabetic patients have high rate of wound infection $15(60 \%)$ in comparison with nondiabetic patients 8 (7.5\%) with RR (8.02) CI (3.67-17.42) and also a finding documented by performing logistic regression (table 9) with a $\mathrm{P}$ value of $<0.0001$.

\section{Conclusions}

Obesity with or without metabolic syndrome has no significant association with either intra-operative or postoperative occurrences in patients with open ventral hernia repair provided their medical conditions are well controlled apart from diabetes which had a significant association with wound infection whether it is a part of metabolic syndrome or not. Further study with more patients enrollment is needed for evaluation of association of intra-operative occurrences in patients with metabolic syndrome and having $\mathrm{BMI}<30$. 


\section{References}

[1] Toro A, Stella G, Gueli A, Mannino M, Palermo F, Burrafato G, Di Carlo I Modified Mayo Technique for Ventral Hernia Repair: An Experimental Study. Chirurgia. 2015; 110(6): 5459.

[2] Carlson MA, Ludwig KA, Condon RE. Ventral hernia and other complications of 1,000 midline incisions. South Med J. 1995; 88(4): 450-453.

[3] Sauerland S, Walgenbach M, Habermalz B, Seiler CM, Miserez M. Laparoscopic versus open surgical techniques for ventral or incisional hernia repair. Cochrane Database Syst Rev. 2011; Issue 3. Art. No.: CD007781. DOI: 10.1002/14651858. CD007781. pub2.

[4] Tran BT, Jeong BY, Oh JK. The prevalence trend of metabolic syndrome and its components and risk factors in Korean adults: results from the Korean National Health and Nutrition Examination Survey 2008-2013. PMC Pub Health. 2017; 17(1): 71 .

[5] "BMI Classification". Global Database on Body Mass Index. World Health Organization. 2006. Retrieved July 27, 2012.

[6] Grundy SM, Cleeman JI, Daniels SR, Donato KA, Eckel RH, Franklin BA, Gordon DJ, Krauss RM, Savage PJ, Smith SC, Spertus JA, Costa F. "Diagnosis and management of the metabolic syndrome: an American Heart Association/National Heart, Lung, and Blood Institute Scientific Statement". Circulation. 2005; 112 (17): 2735-52.

[7] Paniagua JA. Nutrition, insulin resistance and dysfunctional adipose tissue determine the different components of metabolic syndrome. World J Diabetes. 2016 15; 7(19): 483514.
[8] Ford KC, Le TV, Wong ND. Increasing prevalence of the metabolic syndrome among U. S. adults. Diabetes care. 2004; 27(10): 2444-9.

[9] Uakritdathikarn T, Chongsuvivat wong V, Geater AF, Vasinanukorn M, Thinchana S, Klayna S. Perioperative desaturation and risk factors in general anesthesia. J med Assoc Thai. 2008; 91(7): 1020-9.

[10] Krpata, D. M., Blatnik, J. A., Novitsky, Y. W., \& Rosen, M. J. Evaluation of high-risk, comorbid patients undergoing open ventral hernia repair with synthetic mesh. Surg. 2013; 153(1): $120-125$.

[11] Huntington C, Gamble J, Blair L, Cox T, Prasad T, Lincourt A, Heniford BT. Quantification of the effect of diabetes mellitus on ventral hernia repair: results from two national registries. Am Surg. 2016; 82(8): 661-671.

[12] Lledó JB, Quesada YS, Gavara IG, Urbaneja JV, Tatay FC, Diana SB \& Pallardó JM. Prosthetic infection after hernioplasty. Five years experiences. Cirugía Española (English Edition). 2009; 85(3): 158-164.

[13] Fekkes JF, and Velanovich V. "Amelioration of the effects of obesity on short-term postoperative complications of laparoscopic and open ventral hernia repair." Surg laparosc, endosc percutan tech. 2015; 25(2): 151-157.

[14] Robinson TN, Clarke JH, Schoen J, Walsh M D. Major meshrelated complications following hernia repair. Surg Endosc. 2005; 19(12): 1556-1560.

[15] Falagas ME, and Kasiakou SK. Mesh related infections after hernia repair surgery. Clin Microbiol Infect. 2005; 11(1): 3-8.

[16] Wang XC, Zhang D, Yang ZX, Gan JX, \& Yin L. Mesh reinforcement for the prevention of incisional hernia formation: a systematic review and meta-analysis of randomized controlled trials. J Surg Res. 2017; 209: 17-29. 